\title{
The role of intradiscal steroids in the treatment of discogenic low back pain
}

\author{
Stefan Muzin · Zacharia Isaac $\cdot$ Joseph Walker III
}

Published online: 7 December 2007

(C) Humana Press 2007

\begin{abstract}
LBP is one of the most common reasons for visiting a doctor and is the most common cause of disability under age 45.Amongst a variety of etiologies, internal disc disruption (IDD) has been postulated as an important cause of low back pain. Treating discogenic low back pain continues to be a challenge to physicians. Inflammation, either from direct chemical irritation or secondary to an autoimmune response to the nucleus pulposus has been implicated as the primary pain source. Both steroids and non-steroidal anti-inflammatory drugs have partial effectiveness in treating pain associated with inflammation. Therefore, the rationale for using intradiscal steroids is to suppress the inflammation within the disc, thereby alleviating the patient's symptoms. The goal of this article is to review the literature regarding the efficacy of intradiscal steroids to treat low back pain of discogenic origin.
\end{abstract}

Keywords Intradiscal steroids · Hydrocortisone ·

Methylprednisone - Discogenic - Back pain .

Disc disruption $\cdot$ Modic

\section{Introduction}

Low back pain (LBP) remains a major public health concern in the US. LBP is one of the most common reasons for visiting a doctor and is the most common cause of

S. Muzin $(\bowtie) \cdot$ J. Walker III

Department of Physical Medicine and Rehabilitation,

Harvard Medical School, Boston, USA

e-mail: smuzin@gmail.com

Z. Isaac

Brigham and Women's Ambulatory Spine Service, Harvard Medical School, Boston, USA disability under age 45 [1]. Approximately $60-80 \%$ of the US population will experience back pain at some point in their lives and 2-5\% will have it at any given time [2]. According to recent estimates, the annual cost in the US is between 100 and 200 billion dollars.

Amongst a variety of etiologies, internal disc disruption (IDD) has been postulated as an important cause of low back pain [3].

In 1970, Crock invented the term IDD as a condition marked by alteration in the internal structure and metabolic functions of the intervertebral disc, usually proceeded by injuries [4]. He described IDD in a group of patients with low back and leg pain who became worse after surgery for a suspected disc prolapse [5]. Annular tears (including radial and circumferential) are the major form of IDD. It is generally accepted that the diagnosis of discogenic pain due to IDD should meet the following criteria: (1) CT discography reveals an IDD; (2) pain should be reproduced on provocative injection of the contrast, and (3) as a control, stimulation of at least 1 other disc fails to reproduce pain [6].

Discogenic back pain has been defined clinically as chronic low back pain of a deep aching, nagging, or throbbing character, not completely relieved with rest, and sometimes with referred pain to one or both limbs [7]. Treating discogenic low back pain continues to be a challenge to physicians. Over recent years, several minimally invasive treatment modalities such as intradiscal steroid injections, intradiscal electrothermal therapy (IDET), intradiscal radiofrequency (RF) thermocoagulation, and epidural steroid injections have been studied [8].

These methods have triggered a major interest among interventional pain physicians and provide new alternatives for patients with debilitating low back pain [9]. Although the major advantages of minimally invasive therapies 
include their relative simplicity, lower cost, quicker recovery time, and fewer long-term side effects when compared with traditional surgery, many question their efficacy. The goal of this article is to discuss the rationale for using intradiscal steroids and to review the literature regarding the efficacy of intradiscal steroids to treat low back pain of discogenic origin.

\section{Rationale for the use of intradiscal steroids}

The intervertebral disc, which is composed of a central nucleus pulposus and surrounding annulus fibrosis, permits mobility between opposing surfaces of the vertebral body. It is ideally suited for the distribution and dissipation of tremendous longitudinal forces.

The nucleus pulposus, can tolerate a static load indefinitely and adjust to fluctuations through a physiologic response in which there is a compensatory change in fluid retention. Since the nucleus acts as a confined fluid, it distributes vertical forces equally in all directions.

The annulus fibrosus is a highly elastic tissue that surrounds the nucleus pulposus and absorbs tangential forces. With its low matrix content and high concentration of fibrous and elastic tissue, it thrives under tension but deteriorates rapidly when subjected to direct pressure [9].

When degenerative changes exist in the nucleus pulposus, some of the force between the vertebral end plates is exerted directly on the annulus fibrosus. Degeneration of some central fibers appears first, subsequently leading to tears and fissures. Although in the early phases these changes are microscopic in appearance, they cause an inflammatory reaction in the surrounding, sensitive, longitudinal ligaments of the spine. This creates a clinical picture of acute low back pain.

Inflammation, either from direct chemical irritation or secondary to an autoimmune response to the nucleus pulposus has been implicated as the primary pain source [10]. Both steroids and non-steroidal anti-inflammatory drugs have partial effectiveness in treating pain associated with inflammation [11].

When the symptom of pain is thought to result from inflammation, it is natural to think that an anti-inflammatory agent can be useful for treatment. Therefore, the rationale for using intradiscal steroids is to suppress the inflammation within the disc, thereby alleviating the patient's symptoms [12].

Structurally, intradiscal steroids are thought to promote spinal segment stabilization via further disc degeneration. Yong-Hing and Kirkaldy-Willis described the "degenerative cascade" of disc disease [13]. The cascade is divided into three stages based on the amount of damage to the disc and facet joints at a given point in time. This cascade of individual motion segment degeneration is thought of as a continuum rather than as three clearly definable and separate stages.

The first and second stages, dysfunctional and instability stages, respectively, are ongoing fissuring or tearing of the outer annulus fibrosus, decline in the amount of nuclear proteoglycan composition, and patients presenting with intense low back pain [14]. The third stage, stabilization, is characterized by endstage tissue damage and attempts at repair. Nucleus pulposus resorption occurs in this stage resulting in worsening intervertebral disc space narrowing, fibrosis, endplate irregularities, and the formation of osteophytes. The body restabilizes the segment and the patient experiences fewer episodes of back pain [13].

Early studies have shown that after intradiscal injection, radial bulges of the disc are decreased and its height is increased, both are signs of spinal stabilization [15]. Additionally, Kato et al. [16] in 1993 concluded that discograms with intradiscal steroid injections bring about a progressive degeneration of the intervertebral disc. Rather than being a deleterious action, the degeneration of the disc results in clinical improvement (i.e., pain reduction). This occurs because the steroids cause a contraction of the tissue and stabilization of the surrounding spinal segment.

Aoki et al. [17] followed these studies in 1997 and evaluated histologic changes in intervertebral discs of rabbits after intradiscal injection of corticosteroids. They found that after $24 \mathrm{~h}$, rabbits receiving methylprednisolone acetate injection had degenerated tissue in the nucleus pulposus and inner layer of the annulus fibrosus. They also observed matrix vehicles, an indication of primary tissue calcification. They concluded that methylprednisolone causes degeneration and primary calcification in discs. This was proposed as the basis for the clinical improvement in patients where steroids were used within the disc space for the treatment of low back pain and sciatica.

Another practical reason for using intradiscal steroids is that the administration of steroids can be performed at the same time as a diagnostic discogram,without added morbidity, time, or significant expense.

Whether or not intradiscal steroids present a significant risk to patients remains an area of controversy. The risks of spinal canal ossification and calcification are low as concluded by Ito et al. in patients who were followed for 5 years after undergoing discography and betamethasone injection [18]. There have been, however, a few reported cases of necrotic granulomatous lesions associated with intradiscal steroids [19].

While the earlier trials of intradiscal steroids have shown promising potential, more recent trials have been less encouraging. We will review the earlier as well as more recent studies on the use of intradiscal steroid for the treatment of discogenic low back pain. 


\section{Earlier trials}

In 1954, Feffer et al. [9] injected hydrocortisone into the intervertebral discs of 244 patients and followed them for 4-10 years. The population included both patients with and without neurologic deficits, who were deemed good surgical candidates after receiving "extensive conservative care". In most of the cases, discograms were performed by a posterolateral approach in which two interspaces were injected with $25 \mathrm{mg}$ of hydrocortisone. The author attempted to correlate the level treated with the clinical picture. Patients who responded favorably to the injections were placed on a regimen of Williams-type flexion exercises. Most of those who did not respond underwent a laminectomy.

The authors found that 114 of the 244 patients (46.7\%) obtained permanent remission from the hydrocortisone injection. One hundred and thirty patients $(53.3 \%)$ either did not respond to the injection initially or relapsed.

In this study, although no single factor appeared to designate a specifically favorable outcome, older patients (greater than 30 years), patients whose pain was primarily in the back rather than radicular, and those with limited degenerative changes, had the best prognosis. Additionally, the patients' responses could not be predicted from the character or duration of pain.

The only complication noted was one case of interspace infection, which without surgery progressed to an asymptomatic interbody fusion, suggesting faulty sterilization. However, 2-year follow-up roentgenograms were obtained and found "no unusual narrowing or acceleration of the degenerative process."

Wilkinson et al. [20] performed 45 intradiscal injections on 29 patients with lumbar disc disease. Ten of the patients had back pain radiating to the legs and 19 had nonradiating pain. All of the patients had symptoms for a minimum of 6 months, despite aggressive noninvasive therapy. None of the patients were surgical candidates because they had no objective neurological deficits and many had atypical patterns of pain. All patients underwent discography at the time of intradiscal therapy and nearly all of them were abnormal. The discogram abnormalities were generally confined to a single level and correlated closely with the degenerative changes seen on plain film. Early in the series, eight injections were done using 30-40 mg of Depomedrol. Subsequently, all intradiscal injections were done with 60 $80 \mathrm{mg}$ of Depomedrol. All of the patients were followed for a minimum of 1 year, with an average follow-up period of 2.4 years. The authors found that in the group of patients with discogenic pain, 54\% responded poorly and only $31 \%$ had good results lasting for more than 3 months.

In 1960, Leao et al. [21] studied 32 patients aged 18-70, with low back and sciatic pain who failed conservative therapy (rest, lumbosacral corset, and analgesics). Sixteen of the patients received intradiscal hydrocortisone and the remaining received intradiscal prednisolone. The dose of steroid varied from $20-50 \mathrm{mg}$, but the ideal dose was $25 \mathrm{mg}$. The authors measured the patients' response after 24 and $72 \mathrm{~h}$ in both groups as well as after 1 year in the hydrocortisone group. They found that intradiscal steroids produced beneficial results in more than half the patients and that there is no significant difference between intradiscal hydrocortisone and prednisolone.

In 1975, Graham [22] reported a double blind study comparing chemonucleolysis by chymopapain with intradiscal hydrocortisone in a small group of 40 patients who had chronic back pain and sciatica for several years and had failed conservative therapy. Twenty patients were given intradiscal chymopapain injections and 20 were given intradiscal hydrocortisone. The patients were followed for 7 months and then either evaluated by a surgeon who was blinded to the patients' treatment or via questionnaire.

Twelve of the 20 patients $(60 \%)$ receiving chymopapain injections had "fair to good" results after 7 months, and 10 of the 19 patients (53\%) who received hydrocortisone had similar results. They found that chymopapain was not significantly superior to intradiscal injections of hydrocortisone.

This study attempted to prove the superiority of chymopapain over steroids. The authors felt that was unethical to inject placebo (saline) into the disc. Graham concluded "when one is considering the problem of a long-term back sufferer, it is my firm belief that if half of the patients who are subjected to intradiscal injection report back after 7 months and the result has been good, it is certainly a worthwhile procedure [22]."

Although the above studies show that intradiscal steroids may be beneficial when used in select patient populations, they are merely case series and lack an adequate control group. Also, due to their small sample sizes, only limited conclusions may be drawn.

\section{More recent studies}

Due to the lack of objective scientific data in the above studies, more recent trials were done in order to compare intradiscal steroids to intradiscal placebo injections. In 1992, Simmons et al. [23] performed a randomized double blind study to evaluate the efficacy of intradiscal steroid injections when compared with placebo (Bupivicaine). All of the patients selected had only one level disc involvement with or without sciatica and a positive pain response on discography. They all had failed 6 weeks of conservative treatment. Exclusion criteria were multilevel discs, central or lateral stenosis, prior lumbar surgery, or medical disease requiring steroids. A total of 25 patients were randomly 
assigned to receive either Depomedrol $(80 \mathrm{mg} / \mathrm{ml})$ or Bupivicaine (Marcaine) (1.5 ml) and were re-evaluated 10-14 days after the injection. To measure the clinical response, a pain diagram grid score, a visual analog scale, and the Oswestry Pain questionnaire were used before injection and after 10-14 days. Patients with objective significant improvement were entered into the appropriate rehabilitation program, and conservative care was continued. Patients who reported no improvement were considered clinical failures and offered surgery when indicated.

Of the 25 patients studied, 14 received intradiscal Depomedrol and 11 received intradiscal Bupivicaine. In the Depomedrol group, $21 \%$ showed subjective improvement and $79 \%$ showed no improvement. In the Marcaine group, 9\% showed clinical improvement and $91 \%$ showed no improvement. They concluded that there was no statistically significant benefit in using intradiscal steroids.

Khot et al. [7] performed a prospective randomized trial comparing intradiscal steroids to placebo in patients with lumbar discogenic pain. A total of 120 patients with chronic discogenic low back pain who failed at least 6 weeks of conservative care were randomized at the time of discography to either an injection of normal saline or methylprednisolone into the disc space. The patients were followed for 12 months and were asked to report their pain according to a visual analog scale (VAS) and Oswestry Disability Index. The primary outcome measure was the percentage change in their disability. They found that there was no significant difference in the outcome between the two groups as the steroid group had a mean change of $2.28 \%$ disability, while the saline group had a mean percentage change of 3.42 . Also, with respect to pain scores, there was no significant difference.

The results of these two recent studies suggest that intradiscal steroid injections do not improve clinical outcomes in patients with discogenic back pain.

\section{MRI changes after steroid injection}

There have been several studies which suggest that a patient's clinical response to intradiscal steroids can be predicted from their findings on MRI (i.e., Modic changes).

Modic changes on MRI were originally described by Modic in 1988 [24]. MRI type 1 Modic changes are decreased signal on $\mathrm{T} 1$, increased on $\mathrm{T} 2$, usually represent marrow edema, and are associated with an acute process. Type 2 changes, the most common type, are defined as increased signal on $\mathrm{T} 1$ and isointense or slightly hyperintense signal on T2. They represent fatty degeneration of subchondral marrow and are associated with a chronic process. Type 3 changes represent decreased signal on both T1 and T2. It correlates with bony sclerosis (no marrow) on radiographs.
Mayahi et al. [25] sought to determine whether clinical outcome of patients with discogenic back pain who underwent intradiscal steroid injections could be predicted from MRI Modic changes.

Six months after an intradiscal steroid injection, clinical outcomes were measured using visual analog scale and Oswestry disability index. They observed that in patients with Modic 1 vertebral end-plate changes, there was significant relief in $64 \%$ of patients and moderate relief in $29 \%$. In those with Modic 2 changes, there was significant relief in $27 \%$ and moderate relief in $27 \%$. In patients with no Modic changes, there was improvement of low back pain in only $9 \%$. They concluded that patients with Modic 1 changes on MRI are most likely to benefit from intradiscal steroid injections.

Bull et al. [26] studied the imaging of 125 patients who had undergone discography and intradiscal steroid injections for low back pain. They were divided into Non-Modic (61 patients), Type 1 Modic changes (20 patients), and Type 2 Modic changes (24 patients). At 8 weeks postinjection, the patients were assessed and classified as better, the same, or worse. "Better" was defined as complete resolution of symptoms, or significant reduction that allowed return to normal activities.

In the Non-Modic group, 8 of 61 patients (13\%) had improved. In comparison, in the Type 1 Modic group, 4 of the 20 patients $(20 \%)$ improved and in the type 2 Modic group, 13 of the 24 patients $(54 \%)$ were better. From these results, the authors concluded that many patients with Type 2 Modic changes on MRI, may have a chronic inflammatory process which responds well to steroid injections.

More recently, Buttermann [27] studied the effect of epidural and intradiscal steroid injections in patients with degenerative disc disease (DDD), in an attempt to determine whether patients with inflammatory end-plate changes on MRI would respond more favorably to treatment.

Epidural steroid injections (ESI) were performed in 93 patients with degenerative disc disease and inflammatory end-plate changes, and in 139 patients without inflammatory end-plate changes. Additionally, patients with endplate changes (78) and those without changes (93), who were considered candidates for lumbar fusion underwent discography and were randomized as to whether they received intradiscal steroids. Pain and function were prospectively determined by a self-administered outcomes questionnaire that consisted of a visual analog scale, pain drawing, Oswestry Disability Index (ODI), and the patients' opinion regarding treatment success. They were measured before and after the patients' injection for a 2-year followup period.

The authors found that patients with inflammatory endplate changes had greater improvement in the ODI and pain diagram in the first 6 months than those without end-plate 
changes. Intradiscal steroid injections at the time of discography led to significant improvement in patients with inflammatory end-plate changes in all outcome scales but only minimal temporary improvement in patients without end-plate changes. Buttermann concluded that spinal steroid injections are more effective in patients with MRI findings of inflammation, specifically adjacent end-plate changes.

\section{Conclusion}

Based on the current literature, it is unlikely that intradiscal steroids offer much clinical improvement in pain or function for patients with discogenic low back pain. While earlier studies done without control groups have shown encouraging results, more recent controlled trials reveal minimal if any benefit. Recent literature suggests that patients with Modic changes on MRI and signs of end-plate inflammatory changes may benefit the most from intradiscal steroid injections. Research analyzing this subgroup may definitvely determine whether there is a role in this subpopulation. Current literature does not support routine clinical use of intradiscal steroids for the treatment of discogenic low back pain.

\section{References}

1. Woodwell D. National Ambulatory Medical Care Survey: 1996 Summary. Advance Data \#295 1997.

2. Frymoyer JW. Lumbar disk disease: epidemiology. Instr Course Lect 1992;41:217-23.

3. Bogduk N. The lumbar disc and low back pain. Neurosurg Clin $\mathrm{N}$ Am 1991;2:791-806.

4. Crock HV. Internal disc disruption. A challenge to disc prolapse 50 years on. Spine 1986;11:650-3.

5. Crock HV. A reappraisal of intervertebral disc lesions. Med J 1970;1:983-9.

6. Merskey H, Bogduk N. Classification of chronic pain: descriptions of chronic pain syndromes and definitions of pain terms. Seattle: IASP Press;1994. p. 180-1.

7. Khot A, Bowditch M, Powell J, Sharp D. The use of intradiscal steroid therapy for lumbar discogenic pain: a randomized controlled trial. Spine 2004;29:833-6.

8. Zhou Y, Abdi S. Diagnosis and minimally invasive treatment of lumbar discogenic pain. A review of the literature. Clin J Pain 2006;22:468-81.
9. Feffer HL. Therpeutic intradiscal hydrocortisone: a long-term study. Clin Orthop Relat Res 1969;67:100-4.

10. White AA, Gordon SL. Synopsis: workshop on idiopathic lowback pain. Spine 1982;7:141-9.

11. Green LN. Dexamethasone in the management of symptoms due to herniated lumbar disc. J Neurol Neurosurg Psychiatry 1975;38: 1211-7.

12. McCarron RF, Wimpee MW, Hudkins PG, et al. The inflammatory effect of the nucleus pulposus. A possible element in the pathogenesis of low back pain. Spine 1987;12:760-4.

13. Yong-Hing K, Kirkaldy-Willis WH. The pathophysiology of degenerative disease of the lumbar spine. Orthop Clin North Am 1983;14:501-3.

14. Kirkaldy-Willis WH, Farfan HF. Instability of the lumbar spine. Clin Orthop Relat Res 1982;165:110-23.

15. Brinckmann $P$, Horst $M$. The influence of vertebral body fracture, intradiscal injection, and partial discectomy on the radial bulge and height of human lumbar discs. Spine 1985;10(2):138-45.

16. Kato F, Mimatsu K, Kawakami N, et al. Changes in intervertebral disc after discography with intradiscal injection of corticosteroids observed with MRI. J Neurol Orthop Med Surg 1993;14:210-6.

17. Aoki M, Kato F, Mimatsu K, Iwata H. Histologic changes in the intervertebral disc after intradiscal injections of methylprednisolone acetate in rabbits. Spine 1997;22(2):127-31.

18. Ito $\mathrm{S}$, Usui $\mathrm{H}$, Maruyama $\mathrm{K}$, Muro $\mathrm{T}$. Roentgenographic evaluation of ossification and calcification of the lumbar spinal canal after intradiscal betamethasone injection. J Spinal Disord 2001; 14(5):434-8.

19. Menei P, Fournier D, Alhayek G, Mercier P, Francois H, Audran M, Guy G. Rev Rhum Mal Osteoartic 1991;58(9):605-9.

20. Wilkinson HA, Schuman N. Intradiscal corticosteroids in the treatment of lumbar and cervical disc problems. Spine 1980;5: $385-9$.

21. Leao L. Intradiscal injection of hydrocortisone and prednisolone in the treatment of low back pain. Rheumatism 1960;16:72-7.

22. Graham CE. Chemonucleolysis: a preliminary report on a double blind study comparing chemonucleolysis and intradiscal administration of hydrocortisone in the treatment of backache and sciatica. Ortho Clin North Am 1975;6:259-63.

23. Simmons JW, McMillin JN, Emery SF, et al. Intradiscal steroids. A prospective double blind clinical trial. Spine 1992;17:S172-75.

24. Modic MT, Steinberg PM, Ross JS, Masaryk TJ, Carter JR. Degenerative disk disease: assessment of changes in vertebral body marrow with MR imaging. Radiology 1988;166:193-9.

25. Mayahi R, Khot A, Sharp DJ, Powell J. Can modic changes on MRI predict clinical response to steroids in discogenic low back pain? J Bone Joint Surg (Br) 2005;87-B (Supp 3):233.

26. Bull TM, Sharp DJ, Powell JM. The efficacy of intra-discal steroid injection compared to modic changes in degenerate lumbar discs. J Bone Joint Surg (Br) 1998;80-B(1S):47.

27. Buttermann GR. The effect of spinal steroid injections for degenerative disc disease. The Spine J 2004;4:495-505. 